\title{
PERANAN MRI ABDOMEN PADA KASUS HERLYN WERNER WUNDERLICH
}

\author{
Ratna Dessy Witrianti ${ }^{1}$, Nuzul Iman, ${ }^{2}$ Eunike Serfina Fajarini ${ }^{3}$ \\ ${ }^{1-2}$ Departemen Radiologi RS EMC Tangerang \\ ${ }^{3} G E$ Healthcare Indonesia
}

dessyvenus@gmail.com; nuzul_iman@yahoo.com; eunike.fajarini@ge.com

\begin{abstract}
ABSTRACK
Background: Herlyn-Werner-Wunderlich syndrome (HWW) is a very rare genetic syndrome, a combination of anomalies between the mullerian and mesonephric ducts characterized by uterine didelphys, hemivaginal obstruction, and ipsilateral renal agenesis, also known as Obstructed Hemivaginal syndrome and Ipsilateral Renal Anomaly (OHVIRA). Magnetic Resonance Imaging (MRI) is an effective diagnostic support tool for finding out anatomical abdominal abnormalities with the right choice of protocol sequences to obtain optimal MRI images.

Method: This study used a qualitative descriptive approach by presenting a case of a 20 year old female patient who had complaints of lower abdominal pain, no previous medical history and irregular menstruation. MRI Abdomen without contrast was performed using an MRI 1.5 Tesla signa explorer GE Healthcare at EMC Tangerang Hospital. Using the Coronal T2 SSFSE sequence, T1 fat sat LAVA, Axial T2 SSFSE, T1 fat sat LAVA breath hold method and Coronal 3D IFIR (MRA renal without contrast). Specifically for the pelvic area with the Axial T2 Propeller, Coronal T2 fat sat, and Sagittal T2 sequences.

Result: In the Axial T2 propeller sequence, pelvic area retrieval revealed two separate uterine corpuses (uterus didelphys) accompanied by a heterogeneous oval lesion most likely originating from the left side of the vagina (hemivagina). Whereas in the Coronal T2 sequence the whole abdomen was taken and confirmed by the Coronal 3D IFIR, the right kidney was anatomically visible while the left kidney was not visible (agenesis).

Conclusion: Abdominal MRI is a very accurate diagnostic tool to perform Herlyn-WernerWunderlich (HWW) syndrome without contrast agent with SSFSE T2 sequences, T2 propeller and 3D IFIR which have an important role in visualizing the quality of MRI images.
\end{abstract}

Keywords: Herlyn-Werner-Wunderlich ; MRI Abdomen ; very rare

\section{Pendahuluan}

Sindrom Herlyn-Werner-Wunderlich (HWW) merupakan kelainan genetik yang sangat langka, gabungan anomali antara saluran mullerian dan mesonefrik ditandai dengan uterus didelphys, obstruksi hemivagina, dan agenesis ginjal ipsilateral (Hamidi \& Haidary, 2018). Sindrom Herlyn-Werner-Wunderlich dikenal juga dengan sebutan sindrom Obstructed Hemivagina and Ipsilateral Renal Anomaly (OHVIRA) (Girardi Fachin et al., 2019), (Salastekar, Coelho, Majmudar, \& Gupta, 2019) . Etiologi pasti dari sindrom Herlyn-Werner-Wunderlich masih tidak diketahui dan kejadiannya kira-kira $0,1 \%$ hingga 3,8\% (Ekici, Oyardi, Dagistanli, Albayrak, \& Cetin, 2019). Magnetic Resonance Imaging (MRI) abdomen merupakan salah satu alat penunjang diagnostik yang efektif untuk mengetahui kelainan anatomis abdomen dengan pilihan sekuen protokol yang tepat sehingga mendapatkan gambaran atau citra MRI yang optimal untuk menegakkan diagnosa sindrom Herlyn-Werner-Wunderlich (Nishu et al., 2019).

MRI merupakan suatu kombinasi dari sains dan teknologi canggih digital, dan teknologi komputer yang menghasilkan iriisan antomi tubuh secara multiplanar dengan menampilkan jaringan lunak lebih baik daripada CT Scan, sehingga sampai saat ini sebagai salah satu pencitraan pilihan untuk sebagian besar pemeriksaan radiologi (Canellas et al., 2019). 
Pemeriksaan MRI abdomen membutuhkan kerjasama yang baik antara pasien dan radiografer yang bertindak sebagai operator dengan menggunakan metoda teknik breath hold. Teknik breath hold dapat berhasil dengan baik jika pasien dapat mengikuti instruksi dari radiografer karena akan sangat berpengaruh terhadap hasil citra. Pemilihan sekuen yang tepat pada MRI abdomen juga memiliki salah satu peran penting dikarenakan adanya pergerakan di dalam rongga abdomen juga mempertimbangkan untuk mempersingkat waktu pemeriksaan (Del Vescovo et al., 2012).

Pada pengambilan whole abdomen memanfaatkan sekuen T2 Single Shot Fast Spin Echo (SSFSE) atau disebut juga dengan Half Fourier Acquisition Single Shot Turbo Spin Echo (HASTE) dikarenakan sekuen ini dapat mempercepat waktu scanning pada daerah yang bergerak seperti gerakan pada peristaltic usus (Canellas et al., 2019).

Sekuen SSFSE ini didasarkan pada pengisian $k$-space yang tidak lengkap yaitu setengah pengisian dari $k$-space terpenuhi pada satu eksitasi pulsa RF, sedangkan untuk data sisanya dapat dialihkan yang disebut single shot. Istilah single shot ini berari rangkaian pulsa $\mathrm{RF} 180^{\circ}$ yang mengikuti pulsa $90^{\circ}$ dan tidak ada lagi pengulangan pulsa $90^{\circ}$. SSFSE dibandingkan dengan FSE adalah rasio Signal-to-Noise yang berkurang karena waktu akuisisi yang berkurang. (Lingala \& Ghany, 2016).

Teknik propeller dirancang khusus untuk mengurangi efek gerakan alami pada pasien seperti pernapasan, aliran darah, gerakan peristaltic dengan cara melakukan koreksi gerakan secara konsisten sehingga membantu untuk menghasilkan kualitas citra MRI yang lebih baik. Propeller menggunakan pola pengisian $k$-space radial yang menghasilkan peningkatan rasio Signal-to-Noise atau SNR (Communication, 2018).

Untuk pengambilan MRA Renal tanpa kontras dengan menggunakan $3 D$ In Flow Inversion Recovery (IFIR) yang diperlukan untuk penegakkan diagnosa agenesis ginjal ipsilateral. MRA Renal 3D IFIR mampu memvisualisasikan arteri ginjal tanpa menggunakan kontras gadolinium (Schiebler, Reeder, \& Walter, 2015).

\section{Metode}

Penelitian ini menggunakan pendekatan deskriptif kualitatif dengan menyajikan kasus pasien perempuan yang berusia 20 tahun dengan keluhan nyeri perut bagian bawah, tanpa riwayat medis sebelumnya, dan menstruasi yang tidak teratur.

Pemeriksaan MRI Abdomen tanpa kontras dilakukan dengan menggunakan pesawat MRI 1.5 Tesla Signa Explorer GE Healthcare di departemen radiologi RS EMC Tangerang. Protokol MRI Abdomen menggunakan sekuen Coronal T2 SSFSE, T1 fat sat LAVA, Axial T2 SSFSE, T1 fat sat LAVA metoda breath hold dan Coronal 3D IFIR (MRA renal tanpa kontras). Khusus area pelvis dengan sekuen Axial T2 Propeller, Coronal T2 fat sat, dan Sagittal T2.

\section{Hasil dan Pembahasan}

Hasil pemeriksaan MRI abdomen, pada potongan Coronal T2 SSFSE dan Axial T2 propeller memperlihatkan dua corpus uteri yang terpisah (uterus didelphys) disertai lesi heterogen berbentuk oval yang sangat mungkin berasal dari vagina sisi kiri (hemivagina).

Pada 3D IFIR (MRA renal tanpa kontras) tampak anatomis ginjal kanan beserta arterinya sedangkan ginjal kiri dan arteri renalisnya tidak terlihat (agenesis ginjal kiri).

Penulis jelaskan lebih lanjut pada gambar 1, 2 dan 3 sebagai berikut

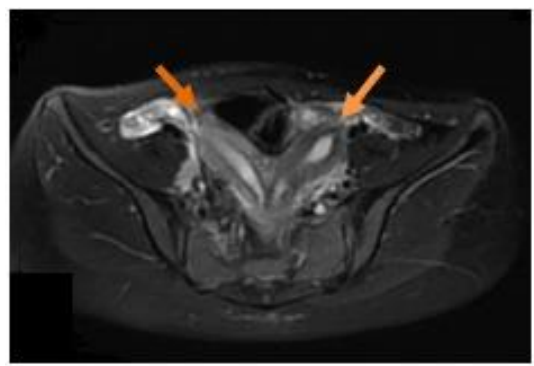

Gambar 1. Axial T2 Propeller (Sumber: RS EMC Tangerang, 2020)

Pada gambar 1. Memperlihatkan dengan jelas dua corpus uteri yang terpisah (uterus didelphys) yang ditunjukkan oleh anak panah orange.
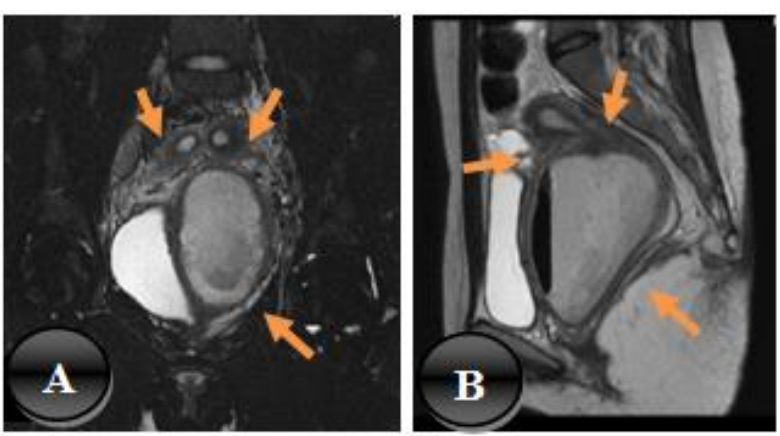
Gambar 2. Coronal T2 Fat Sat (A), Sagital T2 (B) (Sumber: RS EMC Tangerang, 2020)

Pada gambar 2. Tampak dua corpus uteri yang terpisah (uterus didelphys) dan lesi heterogen berbentuk oval yang sangat mungkin berasal dari vagina sisi kiri (hemivagina) yang ditunjukkan oleh anak panah orange.
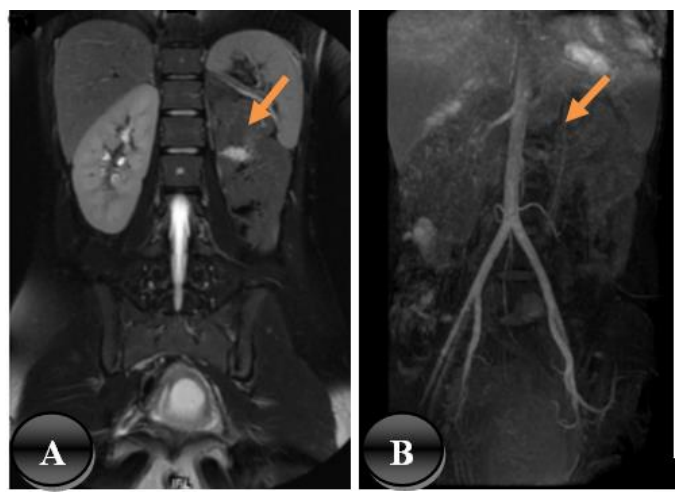

Gambar 3. Coronal T2 (B) dan 3D IFIR (B) (Sumber: RS EMC Tangerang, 2020)

Pada gambar 3. Tampak anatomis ginjal kanan beserta arterinya sedangkan ginjal kiri dan arteri renalisnya tidak terlihat (agenesis ginjal kiri) yang ditunjukkan oleh anak panah orange.

Tabel 1. Parameter MRI Whole Abdomen

\begin{tabular}{lcccc}
\hline \multirow{3}{*}{ Pulse Sequence } & T2 & T1 Fat & T2 & 3D \\
& SSFSE & $\begin{array}{c}\text { Sat } \\
\text { LAVA }\end{array}$ & SSFSE & IFIR \\
\hline Plane & coronal & coronal & axial & coronal \\
\hline
\end{tabular}

\begin{tabular}{lcccc}
\hline TR (ms) & 1000 & 4 & 602 & 4.2 \\
\hline TE (ms) & 62.8 & 1.9 & 97.9 & 2.1 \\
\hline BW & 62.5 & 62.5 & 62.5 & 125 \\
\hline
\end{tabular}

\begin{tabular}{lcccc}
\hline NEX & 1 & 1 & 1 & 1 \\
\hline FOV & $42 \times 42$ & $42 \times 42$ & $42 \times 42$ & $38 \times 34$ \\
\hline Matrix & $288 \times 224$ & $288 \times 192$ & $256 \times 160$ & $224 \times 256$ \\
\hline
\end{tabular}

\begin{tabular}{lccccc} 
Slicelgab $(\mathrm{mm})$ & $5 / 1.5$ & $5 / 1.5$ & $5 / 2.0$ & 2 & m \\
\hline Flip Angle $\left({ }^{\circ}\right)$ & & 12 & & 55 & W \\
\hline Scan time $(\mathrm{s})$ & $00 ; 18$ & $00 ; 17$ & $00 ; 26$ & 2.47 & j \\
\hline Slice & SI & SI & RL & 96 \\
\hline
\end{tabular}

$\begin{array}{llllll}\text { Freqdirection } & \text { SI } & \text { SI } & \text { RL } & \text { SI }\end{array}$

Tabel $1 \mathrm{di}$ atas merupakan parameter dari protokol MRI Whole Abdomen yang dilakukan pada pasien kasus sindrom Herlyn-Werner-Wunderlich (HWW).

Tabel 2. Parameter MRI Pelvic area

\begin{tabular}{lccc}
\hline $\begin{array}{c}\text { Pulse } \\
\text { Sequence }\end{array}$ & $\begin{array}{c}\text { T2 } \\
\text { Propeller }\end{array}$ & T2 FSE & $\begin{array}{c}\text { T2 Fat } \\
\text { Sat }\end{array}$ \\
\hline Plane & axial & sagittal & coronal \\
\hline TR (ms) & 4444 & 2840 & 4664 \\
\hline TE (ms) & 105.4 & 109.8 & 122.5 \\
\hline BW & 41.67 & 135.7 & 141.7 \\
\hline ETL & 30 & 28 & 18 \\
\hline NEX & 2 & 2.5 & 3 \\
\hline FOV & $24 \times 24$ & $20 \times 20$ & $24 \times 24$ \\
\hline Matrix & $288 \times 240$ & $244 \times 244$ & $244 \times 244$ \\
\hline $\begin{array}{l}\text { Slice/gab } \\
\text { (mm) }\end{array}$ & $5 / 1.0$ & $3 / 1.0$ & $3 / 1.0$ \\
\hline Flip Angle $\left(^{\circ}\right)$ & 160 & 160 & 180 \\
\hline Scan time(s) & 3.45 & 2.22 & 3.01 \\
\hline Slice & 34 & 24 & 24 \\
\hline Freq direction & $\mathrm{RL}$ & $\mathrm{AP}$ & $\mathrm{SI}$ \\
\hline
\end{tabular}

Tabel 2 di atas merupakan parameter dari protokol MRI Pelvic area dikhususkan berfokus pengambilan scanning pelvic area sehingga visualisasi uterus beserta organ disekitarnya tampak jelas untuk pasien kasus sindrom HerlynWerner-Wunderlich (HWW) ini.

Pembahasan

Sindrom Herlyn-Werner-Wunderlich (HWW) adalah kasus yang sangat langka antara saluran mullerian dan mesonefrik yang dilaporkan memiliki prevalensi $2 \%-3 \%$ dan insiden 1 dari 200 hingga 1 dari 600 di antara wanita subur. Karakteristik khas dari sindrom ini ditandai dengan adanya uterus didelphys, obstruksi hemivagina, dan agenesis ginjal ipsilateral, sindrom ini dikenal juga dengan istilah OHVIRA (Salastekar et al., 2019), (Tan, Laksmi, Yap, Sadhana, \& Ong, 2020). Sesuai informasi dari jurnal tersebut maka penulis dapat menyampaiakan bahwa kasus sindrom HerlynWerner-Wunderlich (HWW) ini sangat langka dan jarang ada, penulis hanya menemukan satu pasien berusia 20 tahun di bulan maret 2020 .

MRI merupakan pilihan modalitas imajing diagnostik yang dapat menegakkan diagnosis sindrom Herlyn-Werner-Wunderlich dibandingkan dengan modalitas lainnya. Pada penggunaan sequen axial dan sagital T2 menunjukkan dengan jelas uterus didelphys dan adanya obstruksi hemivagina (Mehra, Chamaria, Garga, Kataria, \& Ahuja, 2015). Pada coronal T2 fat sat dan axial T1 juga menunjukkan kelainan uterus didelphys dan 
adanya obstruksi hemivagina (Yadav \& Parmar, 2017). Coronal T2 fat sat menunjukkan agenesis ginjal ipsilateral yang merupakan salah satu tanda sindrom Herlyn-Werner-Wunderlich (HWW) hal ini menunjukkan keunggulan pemeriksaan MRI abdomen tanpa kontras yang sudah dapat memvisualisasikan anatomis ginjal (Yadav \& Parmar, 2017), (Del Vescovo et al., 2012).

Berdasarkan hasil penelitian ini penulis melakukan pemilihan protokol MRI abdomen sesuai dengan standar yang ada dan juga penambahan beberapa sekuen yang memiliki peranan yang vital, seperti SSFSE, propeller, dan juga ditambahkan dengan $3 D$ IFIR. Penggunaan sekuen T2 SSFSE dan T2 propeller terbukti dapat memvisualisasikan citra MRI tanpa ada pergerakan peristalitik perut, serta $3 D$ IFIR mampu menunjukkan kelainan pada arteri renalis tanpa menggunakan kontras.

\section{Simpulan}

MRI abdomen merupakan imajing diagnostik yang sangat akurat dalam memperlihatkan sindrom Herlyn-WernerWunderlich (HWW) tanpa perlu penggunaan kontras dengan sekuen T2 SSFSE, T2 propeller dan $3 D$ IFIR yang memiliki peranan penting dalam memvisualisasikan kualitas citra MRI.

\section{Daftar Pustaka}

Canellas, R., Rosenkrantz, A. B., Taouli, B., Sala, E., Saini, S., Pedrosa, I., ... Sahani, D. V. (2019). Abbreviated MRI protocols for the abdomen. Radiographics, 39(3), 744-758. https://doi.org/10.1148/rg.2019180123

Communication, S. (2018). Short Communication Practical implications of motion correction with motion insensitive radial $\mathrm{k}$-space acquisitions in MRI, (August 2017).

Del Vescovo, R., Battisti, S., Di Paola, V., Piccolo, C. L., Cazzato, R. L., Sansoni, I., ... Zobel, B. B. (2012). Herlyn-werner-wunderlich syndrome: MRI findings, radiological guide (two cases and literature review), and differential diagnosis. BMC Medical Imaging, 12. https://doi.org/10.1186/1471-2342-12-4

Ekici, M. A., Oyardi, P., Dagistanli, F., Albayrak, O., \& Cetin, C. (2019). PEDIATRIC UROLOGY CASE REPORTS, 6(6), 132-136. https://doi.org/10.14534/j-pucr.2019655918
Girardi Fachin, C., Aleixes Sampaio Rocha, J. L., Atuati Maltoni, A., das Chagas Lima, R. L., Arias Zendim, V., Agulham, M. A., ... dos Santos Dias, A. I. B. (2019). Herlyn-Werner-Wunderlich syndrome: Diagnosis and treatment of an atypical case and review of literature. International Journal of Surgery Case Reports, 63, 129-134. https://doi.org/10.1016/j.ijscr.2019.08.035

Hamidi, H., \& Haidary, N. (2018). Late presentation, MR imaging features and surgical treatment of Herlyn-Werner-Wunderlich syndrome (classification 2.2); A case report 11 Medical and Health Sciences 1103 Clinical Sciences. BMC Women's Health, 18(1), 1-4. https://doi.org/10.1186/s12905-018-0655-4

Lingala, S. M., \& Ghany, M. G. M. Mhs. (2016). Increased speed and image quality in single shot fast spin echo imaging via variable refocusing flip angles, 25(3), 289-313. https://doi.org/110.1016/j.bbi.2017.04.008

Mehra, S., Chamaria, K., Garga, U. C., Kataria, A., \& Ahuja, A. (2015). Imaging Diagnosis of HerlynWerner- Wunderlich Syndrome- An Extremely Rare Urogenital Anomaly, 9(5), 10-12. https://doi.org/10.7860/JCDR/2015/11123.5891

Nishu, D. S., Uddin, M. M., Akter, K., Akter, S., Sarmin, M., \& Begum, S. (2019). Herlyn-WernerWunderlich syndrome presenting with dysmenorrhea: A case report. Journal of Medical Case Reports, 13(1), 1-6. https://doi.org/10.1186/s13256-019-2258-6

Salastekar, N., Coelho, M., Majmudar, A., \& Gupta, S. (2019). Herlyn-Werner-Wunderlich syndrome: A rare cause of abdominal pain and dyspareunia. Radiology Case Reports, 14(10), 1297-1300. https://doi.org/10.1016/j.radcr.2019.08.003

Schiebler, M. L., Reeder, S. B., \& Walter, F. (2015). NIH Public Access, 32(2), 190-195. https://doi.org/10.1016/j.mri.2013.10.004.NonContrast

Tan, Y. G., Laksmi, N. K., Yap, T.-L., Sadhana, N., \& Ong, C. C. P. (2020). Preventing the $\mathrm{O}$ in OHVIRA (Obstructed Hemivagina Ipsilateral Renal Agenesis): Early Diagnosis and Management of Asymptomatic Herlyn-WernerWunderlich Syndrome. Journal of Pediatric Surgery, 55(7), 1377-1380. https://doi.org/https://doi.org/10.1016/j.jpedsurg.2 019.06.006

Yadav, T., \& Parmar, P. (2017). Magnetic resonance imaging diagnosis of Herlyn-Werner-Wunderlich syndrome, 172-174. https://doi.org/10.4103/0975-2870.202117 